\title{
Ultrafast All-Optical Switching via Subdiffractional Photonic Nanojets and Select Semiconductor Nanoparticles
}

Brandon Born ${ }^{1, *}$, Simon Geoffroy-Gagnon ${ }^{1}$, Jeffrey D. A. Krupa ${ }^{1}$, Ilija R. Hristovski ${ }^{1}$, Christopher M. Collier ${ }^{1}$, and Jonathan F. Holzman ${ }^{1, *}$

${ }^{1}$ School of Engineering, The University of British Columbia, Okanagan campus, Kelowna, B.C. V1V 1V7, Canada.

Corresponding Authors:

*E-mail (B. Born): brandon.born@ubc.ca

*E-mail (J. F. Holzman): jonathan.holzman@ubc.ca

\begin{abstract}
Nanophotonic all-optical switching is anticipated to replace electronic processing in future optical fibre front-end systems - unlocking capabilities for all-optical terabit-per-second processing. This work introduces a new all-optical switch (AOS) as a fundamental element for such processing. The AOS applies a nanophotonic superlens, in the form of a dielectric microsphere, to form an intense nonevanescent subdiffractional focus called a photonic nanojet. The photonic nanojet materializes in a coating of semiconductor nanoparticles at the rear of the microsphere. The AOS is refined using LorenzMie theory simulations and free-carrier dynamical modelling. Experiments with microspheres coated by $\mathrm{Si}, \mathrm{CdTe}$, InP, and $\mathrm{CuO}$ nanoparticles, having radii of 40, 30, 20, and $20 \mathrm{~nm}$, reveal switching energies of $1 \mathrm{pJ}, 500 \mathrm{fJ}, 400 \mathrm{fJ}$, and $300 \mathrm{fJ}$, and switching times of 2 ps, $2.3 \mathrm{ps}, 900 \mathrm{fs}$, and $350 \mathrm{fs}$, respectively. The realized AOS meets the ultimate goals of femtojoule switching energies and femtosecond switching times.
\end{abstract}

Keywords: nanophotonics, all-optical switching, femtosecond optics, photonics nanojet, semiconductor nanoparticles 
Fibre optic technology has witnessed exponential growth in demand due to its unrivalled data transmission speeds. However, a bandwidth bottleneck ${ }^{1}$ limits these high-speed (terabit-per-second) optical links due to a reliance on far slower (gigabit-per-second) processing by electronic transmitters and receivers. All-optical systems have been proposed to alleviate this bottleneck, and the all-optical switch (AOS) is the fundamental building block of such systems. ${ }^{2,3}$ All-optical switching can ultimately enable terabit-per-second processing in optical fibre front-end systems-in the form of optical time-division multiplexers (OTDM), ${ }^{4}$ orthogonal frequency-division multiplexers (OFDM $),{ }^{5}$ or even network-on-a-chip systems. ${ }^{6,7}$

Nanotechnology has emerged as a key technology to meet the demands of all-optical switching. Nanoscale AOS devices can operate with femtosecond switching times to enable terabit-per-second data rates, and they can operate with femtojoule switching energies to enable future all-optical networks. ${ }^{8-11}$ The demands for switching speed and energy must both be met in a practical AOS implementation, however, and these demands are often mutually exclusive. ${ }^{12-14}$ To develop an AOS that meets both these demands, it is necessary to establish especially strong and rapid interactions between the coincident control (pump) beam and signal (probe) beam.

The onset of the control pulse must initiate strong nonlinear interaction between the control and signal beams to facilitate operation with femtojoule switching energies. As the strength of the interaction is proportional to the intensities, concentrating the beams within a high-intensity focal spot is desirable. Thus, this study introduces a nanophotonic superlens, in the form of a dielectric microsphere, to achieve an intense non-evanescent subdiffractional focus called a photonic nanojet. ${ }^{15-17}$ The microsphere's diameter and refractive index are optimized in this work to maximize the intensity of the photonic nanojet.

The termination of the control pulse must then be followed by rapid recovery of the all-optical switching medium to facilitate operation with femtosecond switching times. With this in mind, we have recently shown that the recovery times can be reduced to femtosecond timescales by using semiconductor nanostructures as the all-optical switching medium. ${ }^{18,19}$ The reduced recovery times, which can be as much as six orders of magnitude lower than those of the bulk semiconductors, result from the prevalence of surface states within the nanostructures. In this study, the semiconductor nanostructures are implemented in the form of semiconductor nanoparticles, of varying compositions. The semiconductor nanoparticles are embedded onto the surface of the aforementioned microsphere. This has the intense focus of the photonic nanojet materialize within the semiconductor nanoparticles.

It is shown that the proposed AOS with integrated photonic nanojets and semiconductor nanoparticles can enable both femtojoule switching energies and femtosecond switching timeswith omnidirectional operation, a small device footprint, and an integrated focusing element. Theoretical models are developed to characterize responses of the photonic nanojets and 
nanoparticles. Experimental results are presented from tests of the developed AOS with semiconductor nanoparticles having varying compositions.

The first point of consideration in this work is the implementation of a nanophotonic superlens with a maximized intensity for its photonic nanojet. An appropriately designed microsphere can establish such a response by refracting incident light at its front surface to an intense focus on its rear surface. The resulting protrusion of intense optical power at and beyond the microsphere's rear surface is referred to as a photonic nanojet. For the purposes of this work, the specific characteristics of a photonic nanojet are defined by the presence of a subwavelength full-width-at-half-maximum (FWHM) and a micron-scale propagation distance. ${ }^{20}$ (It should also be noted that alternative definitions ${ }^{21}$ do exist.) The small focal spot of the photonic nanojet leads to exceedingly high intensities for the interacting control and signal beams - and this can be exploited for reduced switching energies in all-optical applications. The properties of the microsphere must be chosen carefully to attain the ideal characteristics of the photonic nanojet, however, as there exists a complex relationship between the photonic nanojet's intensity and the microsphere's refractive index, $n$, and diameter, $d$. For this work, the refractive index is defined within the range $1.5 \leq n \leq$ 2.0, for realization with contemporary glasses, and the corresponding range of diameters is determined to bring about intense focusing on the microsphere's rear surface. For large spheres, conforming to the thick lens formula, it is known that the highest refractive index in this range, $n \approx$ 2.0 , focuses incident light rays on the microsphere's rear surface. ${ }^{22}$ For smaller spheres, it becomes necessary to carry out three-dimensional electromagnetic analyses, by way of Lorenz-Mie theory simulations, ${ }^{17}$ to define the precise conditions for high-intensity focusing on the microsphere's rear surface.

The results from the Lorenz-Mie theory simulations are shown in Figure 1. The figure shows curves for the intensity at the microsphere's rear surface as a function of its refractive index, $n$. Each data point on the curves is an individual Lorenz-Mie solution. The four displayed curves correspond to microsphere diameters of $d=20 \mu \mathrm{m}, 30 \mu \mathrm{m}, 40 \mu \mathrm{m}$, and $50 \mu \mathrm{m}$. Microspheres with diameters below these values are neglected as there is a desire to avoid resonances within the structure-which come about via longitudinal and/or whispering gallery modes. ${ }^{23}$ The mitigation of such resonances diminishes the effects of cavity lifetimes and enables more rapid all-optical switching times.

It is readily apparent from Figure 1 that microspheres of differing diameters have optimal (peak) intensities at differing refractive indices. For microsphere diameters of $d=20 \mu \mathrm{m}, 30 \mu \mathrm{m}, 40 \mu \mathrm{m}$, and $50 \mu \mathrm{m}$, peak intensities are achieved at the microsphere's rear surface for refractive indices of $n=1.80,1.82,1.83$, and 1.86 , respectively. It is seen here that greater refraction is needed for microspheres of increasing diameters, and this is a result of the interplay between paraxial and nonparaxial focusing. The paraxial region of the beam forms a focal plane farther from the microsphere's centre, in comparison to that formed by the non-paraxial region of the beam. Thus, for the desired 
focusing at the microsphere's rear surface, a large-diameter sphere with a large paraxial region requires a high refractive index, while a small-diameter sphere with a smaller paraxial region requires a low refractive index. In the limiting case of increasing sphere diameters, the refractive index that is required for focusing at the microsphere's rear surface is the geometrical-optics limit of $n \approx 2.0$. For the implementation in this study, a microsphere diameter of $d=40 \mu \mathrm{m}$ is chosen, as its intensity peaks at the refractive index of N-LASF9 glass, $n=1.83$. The photonic nanojet intensity for this microsphere diameter is displayed as the solid black curve in Figure 1.

The intensity profiles at the microsphere's rear surface are shown as insets in Figure 1 for an incident wavelength of $775 \mathrm{~nm}$. Inset 1(a) shows the intensity profile for a refractive index of $n=1.66$. This relatively low refractive index leads to focusing beyond the microsphere and a low intensity at the microsphere's rear surface. Inset 1(b) shows the intensity profile for a refractive index of $n=1.83$. Inset 1(c) shows the intensity profile for a refractive index of $n=2.00$. This relatively high refractive index leads to focusing within the microsphere and a low intensity at the microsphere's rear surface. It can be concluded that a microsphere with a diameter of $d=40 \mu \mathrm{m}$ and refractive index of $n=1.83$ is optimal for the formation of a nanophotonic superlens with a high-intensity photonic nanojet. This refractive index leads to pronounced focusing, with a subwavelength FWHM of $540 \mathrm{~nm}$, being transverse to the optical axis at the microsphere's rear surface, and with a micronscale propagation distance of $1.68 \mu \mathrm{m}$, being the distance along the optical axis over which the beam remains subwavelength. Such characteristics meet the defining criteria for a photonic nanojet. ${ }^{20}$

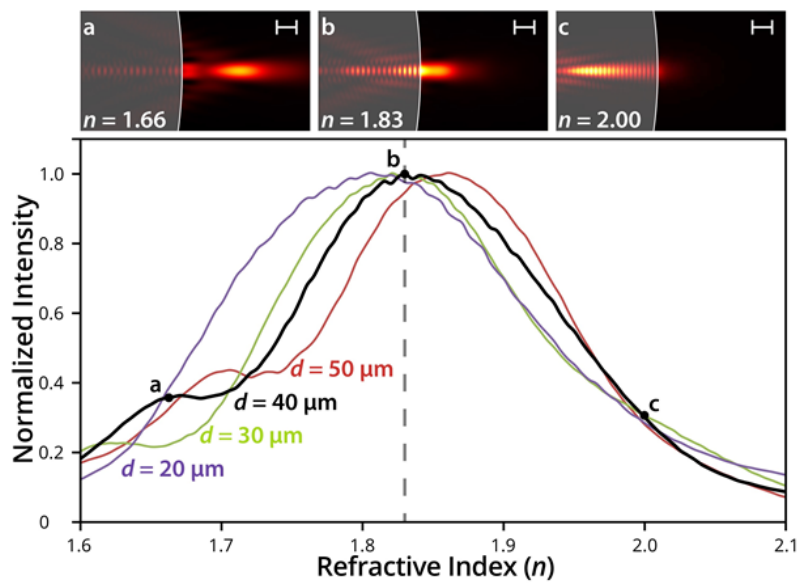

Figure 1. Characterization of photonic nanojet intensities formed by microspheres. Normalized intensity at the microsphere's rear surface is shown versus the microsphere's refractive index, $n$, for microsphere diameters of $d=20 \mu \mathrm{m}$ (purple), $30 \mu \mathrm{m}$ (green), $40 \mu \mathrm{m}$ (black), and $50 \mu \mathrm{m}$ (red). Peak intensities are at $n=1.80,1.82,1.83$, and 1.86 , respectively. Insets 'a', 'b', and 'c' show the intensity at the microsphere's rear surface for $d=40 \mu \mathrm{m}$ and $n=1.66,1.83$, and 2.00, respectively. The results are generated from Lorenz-Mie theory simulations at a wavelength of $775 \mathrm{~nm}$. The scale bar is $1 \mu \mathrm{m}$. 
The second point of consideration in this work is the implementation of semiconductor nanoparticles. The composition and size of the semiconductor nanoparticles must both be considered.

The semiconductor composition is selected with consideration to the nonlinear interaction between the control and signal beams. It is necessary to establish pronounced nonlinear interaction over the micron-scale length of the photonic nanojet, to enable femtojoule switching energies, and this can only be achieved by having a sufficiently strong nonlinearity. With this in mind, semiconductor nanoparticles exhibiting resonant third-order (Kerr) nonlinearities are applied here to mediate interactions between the control (pump) beam at $775 \mathrm{~nm}$ and signal (probe) beam at $1550 \mathrm{~nm}$. This allows the above-bandgap control beam to induce free-carriers and modulate the below-bandgap signal beam over sufficiently short propagation lengths. (Resonant third-order nonlinear coefficients are orders of magnitude higher than their non-resonant counterparts, so the need for phase-matched propagation over millimetre or centimetre lengths ${ }^{3}$ is avoided). In summary, resonant nonlinearity, in the semiconductor nanoparticles, is the means by which the control beam optically switches the signal beam, via free-carrier generation. ${ }^{24}$ The use of resonant nonlinearity, however, leads to an additional constraint, in that the free-carrier lifetime must be made sufficiently short to enable femtosecond switching times.

The nanoparticle size is selected with consideration to surface recombination and its effect on the free-carrier lifetime. Surface recombination comes about through the existence of trapping sites that promote electron-hole recombination. Our prior all-optical switching work has shown that semiconductors in the form of cylindrical nanostructures (photonic crystals) ${ }^{18}$ and spherical nanostructures (nanoparticles) ${ }^{25}$ have especially high surface state densities, and this can lead to rapid surface-assisted recombination and femtosecond switching times.

The nature of the control-signal beam interaction in the semiconductor nanoparticles is revealed by considering the effects of control-beam-induced free-carriers and state-filling on the signal beam. The above-bandgap control beam introduces a free-carrier density, $N(t)$, which decreases the refractive index at the surface (free-carrier dispersion) and increases the absorption in the bulk (freecarrier absorption). ${ }^{26}$ In accordance with Drude theory, these respective responses lead to competing increases and decreases in the signal beam's transmission. Semiconductor nanoparticles do not typically exhibit this competitive response, however, as their scale is far smaller than the optical penetration depth and so surface effects dominate. Thus, nanoparticles predominantly exhibit an increasing signal beam transmission due to free-carrier dispersion. ${ }^{27}$ (It should be noted that the radius of nanoparticles must be well above the Bohr exciton radius to be able to ignore quantum confinement effects - as is the case in this study.) State-filling must also be considered, given that the large surface area of the nanostructures introduces mid-gap surface states within the 
bandstructure. Thus, the free-carriers induced by the control beam can occupy mid-gap surface states and impede the signal beam absorption (occurring to and from these mid-gap surface states) ${ }^{28,29}$ In this study, the differential transmission of the signal beam, $\Delta T(t) / T$, is measured and is seen to be subject to effects of free-carriers and state-filling, with each of these contributions being proportional to the free-carrier density, $N(t)$, within the semiconductor nanoparticles. ${ }^{30}$

A free-carrier dynamical model is formed to develop an understanding of the optical response for a semiconductor nanoparticle. The free-carrier density distribution, $N(r, \theta, t)$, evolves along the radial dimension, $r$, with the polar angle, $\theta$, through time, $t$. The uniformly-illuminated nanoparticles have an initial free-carrier density of $N_{0}$, with these free-carriers undergoing diffusion and recombination in accordance with

$$
\frac{\partial N(r, \theta, t)}{\partial t}=N_{0} \delta(t)+D \nabla^{2} N(r, \theta, t)-\frac{N(r, \theta, t)}{\tau_{\mathrm{b}}},
$$

where the free-carrier photogeneration is quantified by the delta function, $\delta(t)$, diffusion is defined by the diffusion coefficient, $D$, and bulk recombination is defined by the bulk recombination lifetime, $\tau_{\mathrm{b}}$. The diffusing free-carriers ultimately reach the radius, $a$, and undergo surface recombination in accordance with the boundary condition $-D \partial N(r=a, \theta, t) / \partial r=S_{\mathrm{v}} N(r=$ $a, \theta, t)$, where the surface recombination velocity is $S_{\mathrm{v}}$. Once the free-carrier density distribution, $N(r, \theta, t)$, is found, it can be averaged to form the free-carrier density, $N(t)$, and differential transmission of the signal beam, $\Delta T(t) / T$.

For direct solutions to Eq. (1), a frequency-domain (Laplace) approach can be applied. The Laplace solution, $N(r, s)$, is formed, given independence from the polar angle, $\theta$, and its inverse Laplace transform is computed according to

$$
\begin{aligned}
& N(r, t)=\mathcal{L}^{-1}\{N(r, s)\} \\
& =\mathcal{L}^{-1}\left\{\frac{N_{0}}{s+1 / \tau_{\mathrm{b}}}\left[1-\frac{a}{r} \frac{S_{\mathrm{v}} \sinh (k r)}{\left(S_{\mathrm{v}}-D / a\right) \sinh (k a)+D k \cosh (k a)}\right]\right\},
\end{aligned}
$$

where $k=\sqrt{s / D+1 / \tau_{\mathrm{b}}}$. Given the complexity of Eq. (2), a numerical routine is used to compute the inverse Laplace transform, $N(r, t)$, for varying values of the parameters $a, D$, and $S_{\mathrm{v}}$.

For a physical understanding of the solutions to Eq. (1), a time-domain approach can be applied instead. The differential form of Eq. (1) yields a general solution that varies with the radial dimension, $r$, polar angle, $\theta$, and time, $t$. The general solution is ${ }^{31}$

$$
N(r, \theta, t)=\sum_{m=0}^{\infty} \sum_{i=1}^{\infty} \Gamma_{m, i} j_{m}\left(\lambda_{m, i} r / a\right) P_{m}(\cos \theta) \mathrm{e}^{-t / \tau_{m, i}},
$$

where $j_{m}(\cdot)$ and $P_{m}(\cdot)$ are the spherical Bessel functions of the first kind and the Legendre polynomials, respectively, both of order $m$. The eigenvalues, $\lambda_{m, i}$, decay coefficients, $\Gamma_{m, i}$, and time 
constants, $\tau_{m, i}$ are also seen here. The discrete eigenvalues, $\lambda_{m, i}$, are generated by transforming the aforementioned boundary condition into the characteristic equation, $j_{m}\left(\lambda_{m, i}\right) / j_{m}^{\prime}\left(\lambda_{m, i}\right)=$ $-D \lambda_{m, i} /\left(S_{\mathrm{v}} a\right)$, and solving this characteristic equation. The decay coefficients, $\Gamma_{m, i}$, are found by applying the initial condition of a uniform free-carrier density, $N_{0}$. The time constants, $\tau_{m, i}$, are defined by

$$
\frac{1}{\tau_{m, i}}=\frac{1}{\tau_{\mathrm{b}}}+\frac{D \lambda_{m, i}^{2}}{a^{2}}
$$

For the present study, the nanoparticle is uniformly illuminated, with $\theta$ independence, and the nanoparticle radius is much less than the diffusion length. Thus, the general solution in Eq. (3) has a finite term only for $m=0$, and the $i=1$ term of the remaining expansion dominates over the subsequent terms. This allows Eq. (3) to be rewritten as

$$
N(r, t) \approx \Gamma \frac{\sin (\lambda r / a)}{\lambda r / a} \mathrm{e}^{-t / \tau}
$$

where the eigenvalue is $\lambda=\lambda_{0,1}$, the decay coefficient is $\Gamma=\Gamma_{0,1}=2 N_{0}[\sin (\lambda)-\lambda \cos (\lambda)] /[\lambda-$ $\sin (\lambda) \cos (\lambda)]$, and the time constant is $\tau=\tau_{0,1}$. With these definitions, the characteristic equation can be recast in the simplified form of $\tan \lambda / \lambda=(1-S a / D)^{-1}$. The transcendental form of the simplified characteristic equation can be accommodated by computing $\lambda$ with a numerical routine and applying this eigenvalue in Eq. (5) to compute the free-carrier density distribution, $N(r, t)$.

It is worth noting that the transcendental form of the simplified characteristic equation can also be accommodated with further approximations. For a nanoparticle radius that is much less than the diffusion length, i.e., a large diffusion coefficient, $D \gg S_{\mathrm{v}} a$, the eigenvalue will become small, i.e., $\lambda \rightarrow 0$. This allows the left and right sides of the simplified characteristic equation to be expanded, yielding $D \lambda^{2} \approx 3 S_{\mathrm{v}} a$. With this result, Eq. (4) can be rewritten as the generalized approximation of

$$
\frac{1}{\tau} \approx \frac{1}{\tau_{\mathrm{b}}}+S_{\mathrm{v}} R
$$

where $R=3 / a$ is the surface-to-volume ratio of the sphere. The expression in Eq. 6 is the form that is often seen and applied in the literature. ${ }^{32}$ In this limiting case of small $\lambda$, the decay coefficient becomes $\Gamma \approx N_{0}$, the cardinal sine term in Eq. (5) becomes $\sin (\lambda r / a) /(\lambda r / a) \approx 1$, and the freecarrier density distribution simply manifests itself as the average free-carrier density,

$$
N(t) \approx N_{0} \mathrm{e}^{-t / \tau}
$$

The time-domain solutions from Eqs. (4) and (5) are computed for a variety of parameters and are compared to the frequency-domain solutions from Eq. (2). It is found that the solutions match within $1 \%$ error.

The overall responses for time constants of semiconductor nanoparticles are illustrated in Figure 2. The figure shows the time constant, $\tau$, as a function of the surface recombination velocity, $S_{\mathrm{v}}$, and 7 
radius, $a$, for a nanoparticle having a large diffusion coefficient, i.e., $D \gg S_{\mathrm{v}} a$, and long bulk lifetime, i.e., $\tau_{\mathrm{b}} \rightarrow \infty$. It is immediately apparent from this figure that $\tau$ decreases linearly with both $a$ and $1 / S_{\mathrm{v}}$, which is in agreement with the generalized approximation in Eq. (5). Such scaling is of importance in the application of these nanoparticles to all-optical switching, as the observed time constants manifest themselves in the AOS implementations. The red region of Figure 2 is demarcated to show the regime for which femtosecond switching times are achieved. From the bounds of this red region, it is concluded that the semiconductor nanoparticle composition should be selected to have a sufficiently high surface recombination velocity, being greater than $S_{\mathrm{v}}=2000$ $\mathrm{m} / \mathrm{s}$, and the semiconductor nanoparticle size should be selected to have a sufficiently small radius, being less than $a=30 \mathrm{~nm}$. At the same time, the diffusion coefficient of the semiconductor nanoparticle should be sufficiently high, i.e., $D \gg S_{\mathrm{v}}$ a, to maintain rapid diffusive transport of freecarriers from the interior to the surface. If the diffusive transport is impeded by a low diffusion coefficient, i.e., $D \approx S_{\mathrm{v}} a$, the recombination of free-carriers witnesses a bottleneck. This lowdiffusion scenario is illustrated in inset 'a' of Figure 2, which shows the free-carrier density distribution within the semiconductor nanoparticle as a colourmap for $D \approx S_{\mathrm{v}} a$ and a time of 0.5 ps. The colourmap is nonuniform because there is a bottleneck of free-carriers within the interior of the semiconductor nanoparticle. In contrast, if the diffusion coefficient is high, i.e., $D \gg S_{\mathrm{v}} a$, the diffusive transport is sufficiently rapid to have the recombination of free-carriers be readily facilitated by surface states-leading to a relatively uniform free-carrier density distribution within the semiconductor nanoparticle. This high-diffusion scenario is illustrated in inset ' $b$ ' of Figure 2. The free-carrier density distribution within the semiconductor nanoparticle, for $D \gg S_{\mathrm{v}} a$ and a time of $0.5 \mathrm{ps}$, is seen to be a uniform colourmap. In general, it is advantageous to have the diffusion coefficient be as large as possible for rapid diffusive transport.

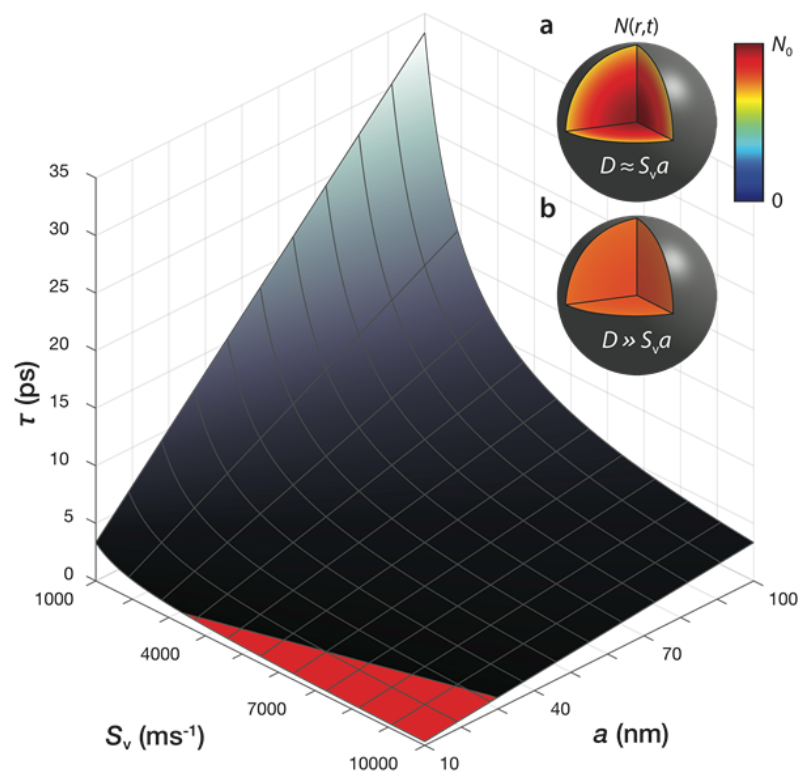


Figure 2. Characterization of time constants for semiconductor nanoparticles. The time constant, $\tau$, i.e., switching time, is displayed versus the surface recombination velocity, $S_{\mathrm{v}}$, and radius, $a$, for a nanoparticle with rapid diffusion, i.e., $D \gg S_{\mathrm{v}} a$ and a long bulk lifetime, i.e., $\tau_{\mathrm{b}}$. Results are formed by solving Eqs. (4) and (5). The red region is the desired (subpicosecond) regime. Inset 'a' shows the nonuniform free-carrier density distribution, $N(r, t=0.5 \mathrm{ps})$, in the nanoparticle for a small diffusion coefficient, i.e., $D \approx S_{\mathrm{v}} a$. Inset 'b' shows the uniform free-carrier density distribution, $N(r, t=0.5 \mathrm{ps})$, in the nanoparticle for a large diffusion coefficient, i.e., $D \gg S_{\mathrm{v}} a$.

The proposed AOS, integrating the photonic nanojet with semiconductor nanoparticles, is tested with a series of experiments. The applied microspheres are made of N-LASF9 glass having a diameter of $d=40 \mu \mathrm{m}$ and refractive index of $n=1.83$. The semiconductor nanoparticles are embedded onto the surface of the microsphere with a dry-coating process. ${ }^{33}$ Van der Waals forces in this process provide sufficient adhesion.

The type of semiconductor used for the semiconductor nanoparticles is constrained by the control and signal beams' wavelengths. The signal beam's wavelength is chosen to be at the telecommunications wavelength of $1550 \mathrm{~nm}(0.8 \mathrm{eV})$ for practical integration, low propagation losses, and increased sensitivity to free-carrier dispersion (which scales with the wavelengthsquared $)^{30}$ The control beam is generated at a wavelength of $775 \mathrm{~nm}(1.6 \mathrm{eV})$, by second harmonic conversion of the signal beam. Based on these two wavelengths, the semiconductor bandgap must fall in the range $0.8 \mathrm{eV}-1.6 \mathrm{eV}$. Si (having a $1.11 \mathrm{eV}$ indirect bandgap), CdTe (having a $1.56 \mathrm{eV}$ direct bandgap), InP (having a $1.27 \mathrm{eV}$ direct bandgap), and p-type $\mathrm{CuO}$ (having a $1.21 \mathrm{eV}$ indirect bandgap $)^{34}$ are selected, as their bandgaps are within the desired range.

The investigations are carried out with a 775-1550 nm pump-probe experimental setup to characterize the impulse response of the AOS. An erbium-doped fibre laser (Toptica Photonics, Inc.) with a 100 fs pulse duration and $90 \mathrm{MHz}$ repetition rate is used. The differential transmission, $\Delta T(t) / T$, of the signal beam is measured as a function of the time-delay the control (pump) and signal (probe) pulses, using a lock-in amplifier (Stanford Research Systems, SR830) with a $100 \mathrm{~ms}$ time constant.

Representative all-optical switching results for each semiconductor are shown in Figure 3, as measurements of the differential signal beam's transmission, $\Delta T(t) / T$. The results for the $\mathrm{Si}, \mathrm{CdTe}$, $\mathrm{InP}$, and $\mathrm{CuO}$ nanoparticles are given in Figure 3(a), (b), (c), and (d), respectively. The minimum switching energies, defined for a signal-to-noise ratio of one, are estimated based on the relative strength of each waveform while the signal and control beam powers are held constant. The switching time (or time constant) is measured via curve-fitting from each $\Delta T(t) / T$ waveform.

Si nanoparticles, shown in the inset of Figure 3(a), have an average radius of $40 \mathrm{~nm}$. The nanoparticles yield a switching energy of $1 \mathrm{pJ}$ and switching time of $1.8 \mathrm{ps}$. The large (picojoule) 
switching energy is attributed to the indirect bandgap of $\mathrm{Si}$ and the resulting poor quantum efficiency. The long (picosecond) switching time is attributed to the large radius of the $\mathrm{Si}$ nanoparticles. The applied free-carrier dynamical model suggests that the Si nanoparticles have a surface recombination velocity of $7400 \mathrm{~ms}^{-1}$. Note that Si nanoparticles would support the desired femtosecond operation, in the red region of Figure 2, given a smaller radius, but such changes would not address the unacceptably high switching energy.

CdTe nanoparticles, shown in the inset of Figure 3(b), have an average radius of $30 \mathrm{~nm}$. The nanoparticles yield a switching energy of $500 \mathrm{fJ}$ and switching time of $2.3 \mathrm{ps}$. The femtojoule switching energy seen here is lower than that of $\mathrm{Si}$, which is attributed to the direct bandgap of CdTe and the correspondingly high quantum efficiency. The picosecond switching time for CdTe is slower than that of Si, however, which implies that the CdTe surface recombination velocity is lower than that of Si. The applied free-carrier dynamical model suggests that the CdTe nanoparticles have a surface recombination velocity of $4300 \mathrm{~ms}^{-1}$. With this surface recombination velocity, the radius could be reduced to enable the desired femtosecond operation in the red region of Figure 2. However, the radius would need to be reduced below $13 \mathrm{~nm}$, and this would encroach upon CdTe's Bohr radius of $7.3 \mathrm{~nm}$. The resulting quantum confinement would likely increase the bandgap beyond the $1.6 \mathrm{eV}$ photon energy of the control beam. ${ }^{35}$

InP nanoparticles, shown in the inset of Figure 3(c), have an average radius of $20 \mathrm{~nm}$. The nanoparticles yield a switching energy of $400 \mathrm{fJ}$ and switching time of $900 \mathrm{fs}$. The femtojoule switching energy seen here for InP is attributed to its direct bandgap. At the same time, the femtosecond switching time seen here is the result of its high surface recombination velocity. The applied free-carrier dynamical model suggests that the InP nanoparticles have a surface recombination velocity of $7400 \mathrm{~ms}^{-1}$. This value, along with the radius, does establish operation in the red region of Figure 2. Thus, InP nanoparticles can meet the concurrent demands for femtojoule switching energies and femtosecond switching times.

$\mathrm{CuO}$ nanoparticles, shown in the inset of Figure 3(d), have an average radius of $20 \mathrm{~nm}$. The nanoparticles yield a switching energy of $300 \mathrm{fJ}$ and switching time of $350 \mathrm{fs}$. The switching energy seen here is especially low. The switching time is the fastest seen, but it is important to note that the recovery exhibits two distinct time constants. We propose that the two time constants are the result of free-carrier recombination, which evolves according to the above analyses, as well as free-carrier relaxation, which is seen through state-filling. The $\mathrm{CuO}$ nanoparticles have a p-type lattice and numerous mid-gap surface states that can act as traps. For such a system, the signal beam undergoes absorption from valence band states to mid-gap states, while the control beam undergoes absorption from valence band states to conduction band states. Thus, the arrival of the control beam depletes the electron occupancy in the valence band, i.e., increases the hole occupancy, which leads to statefilling effects on the signal beam. The signal beam responds with a rapid increase in transmission 
then a rapid decrease in transmission over the $350 \mathrm{fs}$ time constant as holes relax throughout the valence band states. The $350 \mathrm{fs}$ time constant seen for these $\mathrm{CuO}$ nanoparticles is in good agreement with the $400 \mathrm{fs}$ time constant recently measured for relaxation in the valence band of $\mathrm{CuO}$ nanowires. ${ }^{28}$ The relaxed free-carriers then undergo trap-assisted recombination, ${ }^{36}$ which is seen as a weaker $(8 \%)$ decay over a time constant of $2.0 \mathrm{ps}$. (The two time constants were curve fit to be within an R-squared of 0.9997.) Given that the $\mathrm{CuO}$ response is dominated by the femtosecond contributions of state-filling, $\mathrm{CuO}$ nanoparticles can meet the concurrent demands for femtojoule switching energies and femtosecond switching times.

The authors note that the semiconductor nanoparticles employed in this investigation have sufficiently high standard deviations to allow for testing of nanoparticles with varying sizes. The results presented above for $\mathrm{Si}, \mathrm{CdTe}$, and $\mathrm{InP}$ were chosen to have the smallest particles and the shortest time constants. It is interesting to note, however, that the $350 \mathrm{fs}$ time constant of the $\mathrm{CuO}$ nanoparticles was largely independent of the particle size - which supports the interpretation on the predominance of free-carrier relaxation seen via state-filling. In addition, the surface combination velocities quoted here are marginally higher those seen in the literature. ${ }^{18,37,38} \mathrm{We}$ attribute this distinction to the high curvature of the nanoparticles, which leads to pronounced lattice defects and hastened surface recombination, compared to surfaces on a more macroscopic scale.
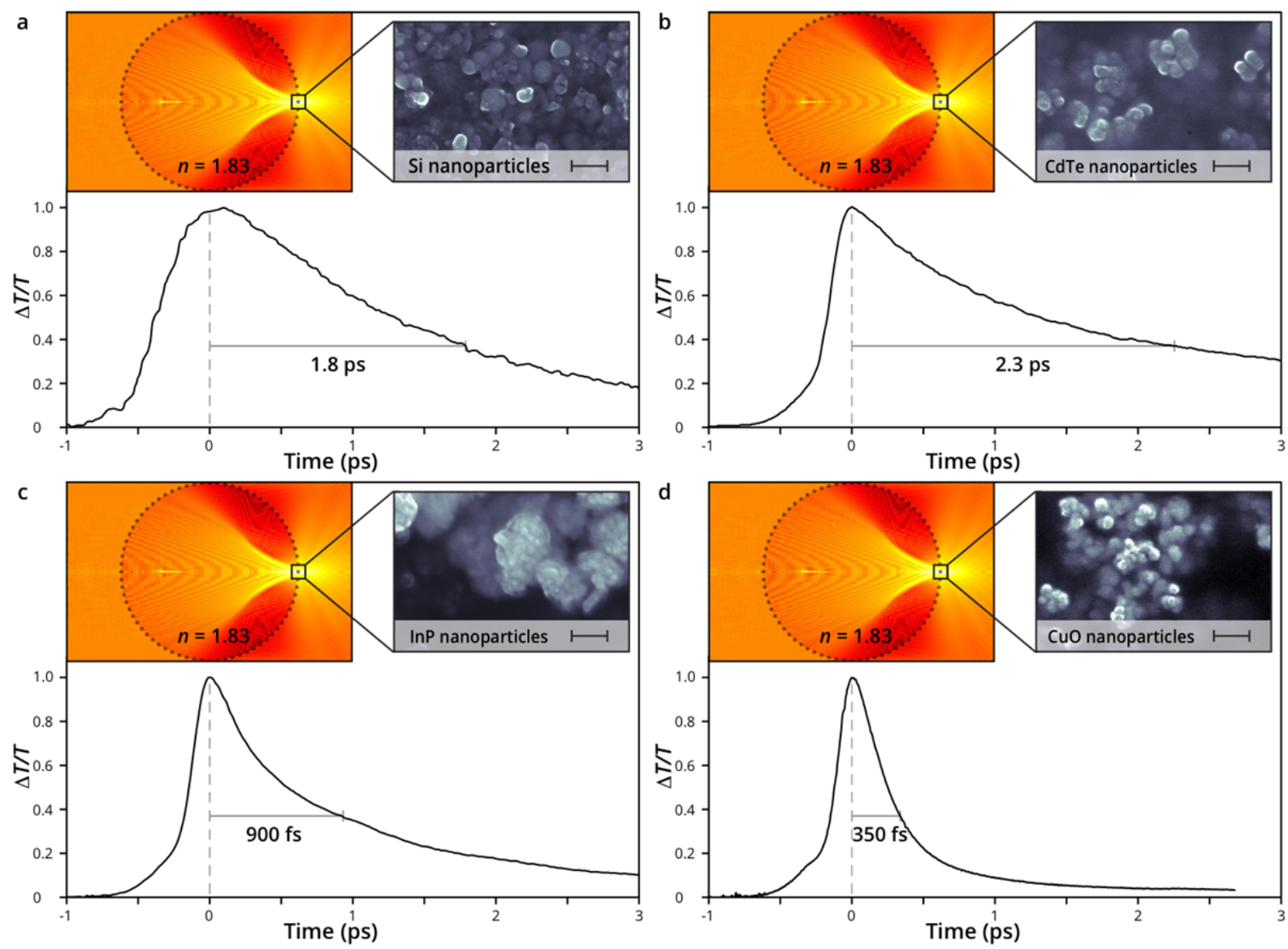
Figure 3. Characterization of the AOS for microspheres with a diameter of $d=40 \mu \mathrm{m}$ and refractive index of $n=1.83$, with select nanoparticle coatings. Normalized differential transmission of the signal beam, $\Delta T / T$, is shown versus time for (a) $\mathrm{Si}$, (b) $\mathrm{CdTe}$, (c) InP, and (d) $\mathrm{CuO}$ nanoparticles. The results exhibit minimum switching energies of 1 pJ, 500 fJ, $400 \mathrm{fJ}$, and $300 \mathrm{fJ}$, with switching times of 2 ps, 2.3 ps, $900 \mathrm{fs}$, and $350 \mathrm{fs}$, respectively. Insets show Lorenz-Mie theory simulations with logarithmic intensities and SEM images of nanoparticles, with a $200 \mathrm{~nm}$ scale bar.

In conclusion, this study investigated the integration of subdiffractional photonic nanojets with semiconductor nanoparticles of select compositions for application to all-optical switching. The characteristics of femtojoule switching energies and femtosecond switching times were pursued. For operation with low switching energies, a nanophotonic superlens was applied in the form of a dielectric microsphere. The appropriately designed dielectric microsphere was found to establish a photonic nanojet at its rear surface with an especially high intensity This yielded enhanced interaction between the control and signal beams. Optimal characteristics were defined by LorenzMie theory simulations, leading to the selection of an N-LASF9 glass microsphere with a diameter of $d=40 \mu \mathrm{m}$ and refractive index of $n=1.83$. For operation with short switching times, semiconductor nanoparticles were applied on the microsphere's rear surface. The semiconductor nanoparticles were comprised of $\mathrm{Si}, \mathrm{CdTe}, \mathrm{InP}$, and $\mathrm{CuO}$. It was found that the increased presence of surface states within the semiconductor nanoparticles led to hastened surface recombination and rapid recovery. A free-carrier dynamical model was developed to reveal a deeper understanding (and exploitation) of this recovery. The AOS, employing photonic nanojets with $\mathrm{Si}, \mathrm{CdTe}$, InP, and $\mathrm{CuO}$ nanoparticles, was tested. It was found to have minimum switching energies, estimated to be $1 \mathrm{pJ}$, $500 \mathrm{fJ}, 400 \mathrm{fJ}$, and $300 \mathrm{fJ}$, and switching times, being 2 ps, 2.3 ps, $900 \mathrm{fs}$, and $350 \mathrm{fs}$, respectively. The optimal semiconductor witnessed in this study was $\mathrm{CuO}$, which readily achieved the goals of femtojoule switching energies and femtosecond switching times.

The authors also note that the proposed AOS can meet the performance demands of emerging optical fibre front-end operations, such as optical multiplexing. ${ }^{3,5}$ At the same time, from a practical standpoint, the monolithic architecture of the AOS, with a compact device footprint and an integrated focusing element, allows for ease of coupling and omnidirectional operation. Such an architecture can be readily applied in cascaded on-chip implementations, through daisy-chained spheres, ${ }^{39}$ which have proven to be challenging ${ }^{40}$ for AOS devices. Future work could improve upon the AOS by tuning the nanoparticles for Mie resonance to increase the modulation depth. ${ }^{41,42}$ Overall, the proposed AOS can become a fundamental element in future all-optical implementations.

\section{Acknowledgement}


This work was supported by the Natural Sciences and Engineering Research Council of Canada (341487-12), and the Canada Foundation for Innovation (LOF 16659). We thank D. J. Arkinstall and the Charles E. Fipke Foundation for use of the STAR SEMLab at the University of British Columbia’s Okanagan Campus.

\section{References}

1. Li, F.; Vo, T. D.; Husko, C.; Pelusi, M.; Xu, D.-X.; Densmore, A.; Ma, R.; Janz, S.; Eggleton, B. J.; Moss, D. J. All-Optical XOR Logic Gate for 40Gb/s DPSK Signals via FWM in a Silicon Nanowire. Opt. Express 2011, 19, 20364-20371.

2. Brzozowski, L.; Sargent, E. H. All-Optical Analog-to-Digital Converters, Hardlimiters, and Logic gates. J. Lightwave Technol. 2001, 19(1), 114-119.

3. Nazarathy, M.; Zalevsky, Z.; Rudnitsky, A.; Larom, B.; Nevet, A.; Orenstein, M.; Fischer, B. All-Optical Linear Reconfigurable Logic with Nonlinear Phase Erasure. J. Opt. Soc. Am. 2009, 26, 21-39.

4. Vo, T. D.; Hu, H.; Galili, M.; Palushani, E.; Xu, J.; Oxenløwe, L. K.; Madden, S. J.; Choi, D.Y.; Bulla, D. A. P.; Pelusi M. D.; Schröder, J.; Luther-Davies, B.; Eggleton, B.J. Photonic Chip Based Transmitter Optimization and Receiver Demultiplexing of a $1.28 \mathrm{Tbit} / \mathrm{s}$ OTDM Signal. Opt. Express 2010, 18, 17252-17261.

5. Hillerkuss, D.; Schmogrow, R.; Schellinger, T.; Jordan, M.; Winter, M.; Huber, G.; Vallaitis, T.; Bonk, R.; Kleinow, P.; Frey, F.; Roeger, M.; Koenig, S.; Ludwig, A.; Marculescu, A.; Li, J.; Hoh, M.; Dreshmann, M.; Meyer, J.; Ben Ezra, S.; Nariss, N.; Nebendahl, B.; Parmigiani, F.; Petropoulos, P.; Resan, B.; Oehler, A; Weingarten, L.; Ellermeyer, T.; Lutz, J.; Moeller, M.; Huebner, M.; Becker, J.; Koos, C.; Freude, W.; Leuthold, J. 26 Tbit s $^{-1}$ Line-Rate SuperChannel Transmission Utilizing All-Optical Fast Fourier Transform Processing. Nature Photon. 2011, 5, 364-371.

6. Shacham, A.; Bergman, K.; Carloni, L. P. On the Design of a Photonic Network-on-Chip. Proc. of the First International Symposium on Networks-on-Chip. 2007, 2053-64.

7. Hao, Hu; Hua, Ji; Minhao, Pu; Galili, M.; Yvind, K.; Oxenlowe, L.K. 160-Gb/s Silicon AllOptical Packet Switch for Buffer-less Optical Burst Switching. J. Lightwave Technol., 2015, 33(4), 843-848.

8. Miller, D. A. Device Requirements for Optical Interconnects to Silicon Chips. Proc. IEEE 2009, 97, 1166-1185.

9. Husko, C.; De Rossi, A.; Combrie, S.; Tran, Q. V.; Raineri, F.; Wong, C. W. Ultrafast AllOptical Modulation in GaAs Photonic Crystal Cavities. Appl. Phys. Lett. 2009, 94, 021111. 
10. Hu, X.; Jiang, P.; Ding, C.; Yang, H.; Gong, Q. Picosecond and Low-Power All-Optical Switching Based on an Organic Photonic-Bandgap Microcavity. Nat. Photon. 2008, 2, 185189.

11. Volz, T.; Reinhard, A.; Winger, M.; Badolato, A.; Hennessy, K. J.; Hu E. L.; Imamoglu, A. Ultrafast all-optical switching by single photons. Nature Photon. 2012, 6, 607-611.

12. Gupta, S.; Frankel, M. Y.; Valdmanis, J. A.; Whitaker, J. F.; Mourou, G. A.; Smith, F. W.; Calawa A. R. Subpicosecond Carrier Lifetime in GaAs Grown by Molecular Beam Epitaxy at Low Temperatures. Appl. Phys. Lett. 1991, 59, 3276-3278.

13. Almeida, V. R.; Barrios, C. A.; Panepucci, R. R.; Lipson, M. All-Optical Control of Light on a Silicon Chip. Nature 2004, 431, 1081-1084.

14. Nozaki, K.; Tanabe, T.; Shinya, A.; Matsuo, S.; Sato, T.; Taniyama, H.; Notomi, M. Subfemtojoule All-Optical Switching Using a Photonic-Crystal Nanocavity. Nature Photon. 2010, 4, 477-483.

15. Chen, Z. G.; Taflove, A.; Backman, V. Photonic Nanojet Enhancement of Backscattering of Light by Nanoparticles: A Potential Novel Visible-Light Ultramicroscopy Technique. Opt. Express 2004, 12, 1214-1220.

16. Li, X.; Chen, Z.; Taflove, A.; Backman, V. Optical Analysis of Nanoparticles via Enhanced Backscattering Facilitated by 3-D Photonic Nanojets Opt. Express 2005, 13, 526-533.

17. Lecler, S.; Takakura, Y.; Meyrueis, P. Properties of a Three-Dimensional Photonic Jet. Opt. Lett. 2005, 30, 2641-2643.

18. Holzman, J. F.; Strasser, P.; Wuest, R.; Robin, F.; Erni D.; Jackel H.; Ultrafast Carrier Dynamics in InP Photonic Crystals. Nanotechnol. 2005, 16, 949-952.

19. Born, B.; Krupa, J. D. A.; Geoffroy-Gagnon, S.; \& Holzman, J. F. Integration of Photonic Nanojets and Semiconductor Nanoparticles for Enhanced All-Optical Switching, Nat. Comm. 2015, 6, 8097.

20. Ferrand, P.; Wenger, J.; Devilez, A.; Pianta, M.; Stout, B.; Bonod, N.; Popov, E.; Rigneault, H. Direct imaging of photonic nanojet. Opt. Express, 2008, 16, 6930-6940.

21. Geints, Y. E.; Zemlyanov, A. A.; Panina, E. K. Control over parameters of photon nanojets of dielectric microspheres. Opt. Commun. 2010, 283, 4775-4781.

22. Welford. W. T. Aberration of Optical Systems. Adam Hilger Ltd.: London, 1986.

23. Geints, Y.; Zemlyanov, A.; Panina, E. Photonic Jets from Resonantly Excited Transparent Dielectric Microspheres. J. Opt. Soc. Am. B. 2012, 29, 758-762.

24. Klimov, V. I. J. Optical Nonlinearities and Ultrafast Carrier Dynamics in Semiconductor Nanocrystals. Phys. Chem. B. 2000, 104, 6112-6123.

25. Collier, C. M.; Born, B.; Holzman, J. F. Ultrafast Response of SiC and Si Nanocomposite Material Systems. Electron. Lett. 2012, 48, 1618-1619. 
26. Collier, C. M.; Born, B.; Bethune-Waddell, M.; Jin, X.; Holzman, J. F. Ultrafast

Photoexcitation and Transient Mobility of $\mathrm{GaP}$ for Photoconductive Terahertz Emission. IEEE J. Quant. Electron. 2013, 49, 691-696.

27. Hamerly, R.; Mabuchi, H. Quantum Noise of Free-carrier Dispersion in Semiconductor Optical Cavities. Phys. Rev. A 2015, 92, 023819.

28. Othonos, A.; Zervos, M. Ultrafast Hole Carrier Relaxation Dynamics in P-Type CuO Nanowires. Nanoscale Res. Lett. 2011, 6, 622-626.

29. Othonos, A.; Zervos, M.; Tsokkou, D. Tin Oxide Nanowires: The Influence of Trap States on Ultrafast Carrier Relaxation. Nanoscale Res. Lett. 2009, 4, 828-833.

30. Grumstrup, E. M.; Cating, E. E.; Gabriel, M. M.; Pinion, C. W.; Christesen, J. D.; Kirschbrown, J. R.; Vallorz, E. L. I.; Cahoon, J. F.; Papanikolas, J. M. Ultrafast Carrier Dynamics of Silicon Nanowire Ensembles: The Impact of Geometrical Heterogeneity on Charge Carrier Lifetime. J. Phys. Chem. C 2014, 118, 8626.

31. Gharghi, M.; Cheng, C.; Sivoththaman, S. Minority Carrier Recombination in Spherical Silicon Crystals: Measurement and Process-Induced Effects. Semicond. Sci. Technol. 2008, 23, 125035.

32. Jurienas, S.; Stepankevieius, V.; Strumskis, M.; Zukauskas, A. Carrier Recombination in CdS Nanocrystals Under Single-Electron and High-Density Excitation. Semicond. Sci. Technol. 2008, 10, 301-309.

33. Borderieux, S.; Wu, C. Y.; Bonzongo, J.-C.; Powers, K. Control of Elemental Mercury Vapor in Combustion Systems using Fe2O3 Nanoparticles. Aerosol Air Qual. Res. 2004, 4, 74-90.

34. Zoolfakar, A. S.; Rani, R. A.; Morfa, A. J.; O’Mullane, A. P.; Kalantar-zadeh, K. Nanostructured Copper Oxide Semiconductors: a Perspective on Materials, Synthesis Methods and Applications. J. Phys. Chem. C. 2014, 2, 5247-5270.

35. Groeneveld, E.; Delerue, C.; Allan, G.; Niquet, Y. M.; Donega, C. D. Size Dependence of the Exciton Transitions in Colloidal CdTe Quantum Dots. J. Phys. Chem. C. 2012, 116, 23160.

36. Linnros, J. Carrier Lifetime Measurements using Free Carrier Absorption Transients. I. Principle and injection dependence. J. Appl. Phys. 1998, 84, 275-283.

37. Kuciauskas, D.; Kanevce, A.; Dippo, P.; Seyedmohammadi, S.; Malik R. Minority-Carrier Lifetime and Surface Recombination Velocity in Single-Crystal CdTe. IEEE J. Photovoltaics 2015, 5, 366-371.

38. Mackel, H.; Cuevas, A. Determination of the Surface Recombination Velocity of Unpassivated Silicon from Spectral Photoconductance Measurements. Proceedings of the Third World Conference on Photovoltaic Energy Conversion. 2003, 71-74.

39. Kapitonov, A. M.; Astratov, V. N. Observation of Nanojet-Induced Modes with Small Propagation Losses in Chains of Coupled Spherical Cavities. Opt. Lett. 2007, 32, 409-411. 
40. Ballarini, D.; De Giorgi, M.; Cancellieri, E.; Houdré, R.; Giacobino, E.; Cingolani, R.;

Bramati, A.; Gigli G.; Sanvitto D. All-Optical Polariton Transistor. Nat. Commun. 2013, 4, 1778.

41. Shcherbakov, M. R.; Vabishchevich, P. P.; Shorokhov, A. S.; Chong, K. E.; Choi, D.-Y.; Staude, I.; Miroshnichenko, A. E.; Neshev, D. N.; Fedyanin, A. A.; Kivshar, Y. S. Ultrafast All-Optical Switching with Magnetic Resonances in Nonlinear Dielectric Nanostructures. Nano Lett. 2015, 15, 6985-6990.

42. Makarov, S.; Kudryashov, S.; Mukhin, I.; Mozharov, A.; Milichko, V.; Krasnok, A.; Belov, P. Tuning of Magnetic Optical Response in a Dielectric Nanoparticle by Ultrafast Photoexcitation of Dense Electron-Hole Plasma. Nano Lett. 2015, 15, 6187. 


\section{FOR TABLE OF CONTENTS USE ONLY}

Manuscript Title: Ultrafast All-Optical Switching via Subdiffractional Photonic Nanojets and Select

Semiconductor Nanoparticles

Names of Authors: Brandon Born, Simon Geoffroy-Gagnon, Jeffrey D. A. Krupa, Ilija R. Hristovski, Christopher M. Collier, and Jonathan F. Holzman

Synopsis: The Table of Contents Graphic depicted below illustrates the general concept of the all-optical switch (AOS) employed in this study. The AOS applies a nanophotonic superlens, in the form of a dielectric microsphere, to form an intense non-evanescent subdiffractional focus called a photonic nanojet. The photonic nanojet materializes in a coating of semiconductor nanoparticles at the rear of the microsphere. A Lorenz-Mie theory simulation on the left depicts the photonic nanojet, and a scanning electron microscope image of $\mathrm{CuO}$ nanoparticles on the right illustrates the coating of semiconductor nanoparticles.

\section{Table of Contents Graphic:}

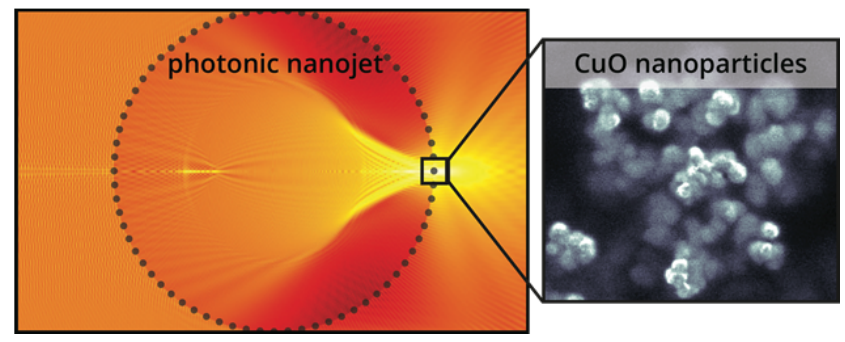

